\title{
Introducción al uso de modelos aplicados de equilibrio general
}

Arturo Pérez, Mendoza*

\section{RESUMEN}

Este trabajo presenta la metodología utilizada para elaborar modelos aplicados de equilibrio general (MEGA). Se presenta un modelo muy sencillo que sirve para ilustrar todos los pasos a seguir para especificar, calibrar y realizar simulaciones con un MEGA diseñado para la evaluación de una política económica. A partir de la lectura de este trabajo el lector que quiera familiarizarse con esta metodología podrá consultar y entender con mayor facilidad la bibliografía escrita sobre este tema.

Número de clasificación: JEL: D58

Palabras clave: equilibrio general, calibración, modelos aplicados, matriz de contabilidad social, variación compensada, variación equivalente.

\begin{abstract}
This work presents the methodology used to construct Applied General Equilibrium Models (MEGAs). It presents a very simple model that can be used to illustrate all the steps needed to specify, calibrate and make simulations using a MEGA designed for the evaluation of an economic policy. From the reading of this work the reader who wants to familiarize with this methodology will be able to consult and to understand with more facility the literature written on this topic.
\end{abstract}

Clasification number: JEL: D58

Keywords: general equilibrium, calibration, applied models, social accounting matrix, compensated variation, equivalent variation.

* Doctor en Economía por la Universidad de Minnesota. Profesor de tiempo completo en el Tecnológico de Monterrey, campus Ciudad de México [arturo.perez@itesm.mx]. 


\section{INTRODUCCIÓN}

Este trabajo presenta de una manera sencilla la metodología para elaborar modelos de equilibrio general aplicados (MEGA). Su objetivo es servir como punto de partida para el investigador que quiera familiarizarse con esta metodología. Por tal motivo, expone y analiza un modelo sencillo que permite mostrar con claridad los pasos a seguir para su aplicación en el análisis de la política económica. Los MEGA han sido utilizados ampliamente para analizar impactos de política económica a partir del trabajo de Johansen (1960).

Diversos artículos han documentado aplicaciones realizadas para evaluar impactos de política comercial, fiscal y energética, entre otras. ${ }^{1}$ En México se han elaborado MEGA para analizar problemas diversos. Algunos ejemplos de modelos elaborados recientemente son los trabajos de Sobarzo (2004), en el que se evalúa una iniciativa de reforma fiscal del gobierno mexicano; Gutiérrez et al. (2005), que examina el impacto de la política fiscal en la administración de los recursos hidráulicos, y Pérez (2006), el cual examina el impacto de imponer una salvaguarda a las importaciones de triplay. En el mundo cada vez más gobiernos e institutos de investigación de política económica utilizan esta herramienta para su análisis. Devarajan y Robinson (2002) reportan que en al menos 20 países los gobiernos e institutos de investigación en política económica saben elaborar este tipo de modelos, en tanto que en muchos otros países los gobiernos suelen contratar a consultores e institutos no gubernamentales para su realización.

Elaborar un MEGA significa convertir la estructura walrasiana de equilibrio general - formalizada en la década de los años cincuenta por Arrow y Debreu-, de un modelo abstracto en un modelo práctico que represente una economía en concreto y que permita evaluar el impacto de una política económica específica. El modelo teórico de equilibrio general plantea una situación en la que todos los agentes de la economía tienen un comportamiento optimizador que genera oferta y demanda de bienes y servicios. Los diferentes precios se mueven para permitir que dicho comportamiento optimizador genere un equilibrio de la oferta y la demanda en todos los mercados. Una posible objeción a este tipo de modelos sería el hecho de que en la práctica podemos observar economías en las que no todos los mercados están en equilibrio, lo cual puede ser cierto sobre todo en el mercado laboral. Sin embargo, ésta no resulta ser una objeción válida si to-

\footnotetext{
${ }^{1}$ Para una comparación de diversos modelos utilizados para evaluar impactos de política fiscal y comercial véase Shoven y Whalley (1984). Este artículo es un clásico en este tema y puede ser consultado también como un artículo introductorio.
} 
mamos en cuenta que el modelo es una representación abstracta de la realidad y que si realmente ese desequilibrio es muy importante para el análisis, entonces se debe de modelar de esa manera, como un mercado en desequilibrio. Por ejemplo, modelos que consideran muy importante el análisis del impacto sobre la tasa de desempleo de un cambio de política, pueden asumir que el mercado de trabajo no está en equilibrio, que el salario es fijo y que los únicos cambios que se presentan en este mercado implican un mayor o menor nivel de empleo.

Un modelo basado en la teoría de equilibrio general permite captar efectos de mejor manera que otro tipo de modelos, ya sea econométricos o de equilibrio parcial. Un MEGA permite analizar de qué manera cambios en política económica generan reasignación de recursos entre sectores, redistribución de ingreso entre consumidores, evaluar pérdidas y ganancias de bienestar, etcétera. A pesar de que algún modelo sea elaborado para evaluar el impacto de una política económica dirigida a un sector en particular, se pueden conocer los impactos generados en otros sectores de manera directa e indirecta, así como otros cambios gracias a la flexibilidad con que se pueden incorporar diversos detalles de la economía en este tipo de modelos.

Parte muy importante para tomar la decisión de utilizar este tipo de modelos radica en saber qué tan precisas son sus predicciones respecto a los posibles impactos que puede generar un cambio de política económica. En este sentido, es importante remarcar que lo acertado de un modelo depende de qué tan bien ha sido construido de acuerdo con el tipo de problema que se quiere analizar. Por ejemplo, un modelo construido para analizar los posibles impactos de una apertura comercial requiere tomar en cuenta la existencia de economías de escala y competencia imperfecta en sectores con estas características, en lugar de suponer que existen rendimientos constantes a escala y competencia perfecta, como es común en muchos modelos. No tomar en cuenta esto puede resultar en un modelo que subestime los verdaderos impactos de una apertura comercial.

Kehoe (2003) evaluó el desempeño de tres de los más importantes MEGA utilizados para predecir el impacto del Tratado de Libre Comercio de América del Norte (TLC) y encontró que los modelos subestiman el impacto de dicho tratado, además de que no permiten generar mucho comercio en aquellos sectores en los que existía poco antes del tratado. Esto, desde luego, no invalida la utilización de este tipo de modelos. Más bien, hace ver que si se quiere utilizar un MEGA para analizar una liberalización comercial semejante, no se puede utilizar el mismo tipo de modelo empleado para prever el impacto del TLC sin antes hacerle los cambios convenientes para que genere mejores predicciones. En ese mismo 
trabajo, Kehoe hace referencia a otro MEGA construido para analizar el impacto sobre la economía española de las reformas implantadas en 1986, necesarias para su ingreso a la entonces llamada Comunidad Europea. El análisis de este modelo muestra que tuvo un buen desempeño en predecir los cambios que en realidad ocurrieron. Estas pruebas permiten aumentar el rigor de este tipo de modelos: “...algunas pruebas establecerán preguntas que sólo pueden ser resueltas con modificaciones en la teoría... Para reforzar que el análisis de equilibrio general aplicado es una disciplina científica, fallas de la teoría pueden ser aun más importantes que confirmaciones de su progreso."2

A pesar de que los MEGA constituyen una herramienta muy útil para evaluar impactos de política económica, su metodología no suele ser enseñada en los programas de licenciatura y posgrado en México. Cuando el investigador desea conocer la forma de elaborar un MEGA puede recurrir a libros y artículos muy útiles, pero que en muchas ocasiones suelen ser poco claros al no entrar al detalle en algunos puntos, o al basar su explicación en modelos muy elaborados compuestos por varios elementos que integran una economía (gobierno, transacciones intersectoriales, ahorro-inversión, diversos consumidores y sector externo). Este trabajo explica la forma en que se puede elaborar un MEGA a partir del uso de un modelo muy sencillo, en el que se representa la economía mediante un consumidor representativo, dos sectores productivos y dos factores de la producción. A partir de la lectura de este trabajo, se pueden consultar otras fuentes que permitirán completar lo aprendido en este artículo. ${ }^{3}$

La figura 1 muestra los pasos a seguir para la elaboración y aplicación de los MEGA. La elaboración de un MEGA surge a partir de un problema que involucra el uso de una política económica. Dependiendo del problema, se realiza la especificación del modelo, es decir, se definen las ecuaciones que lo integrarán. Posteriormente, se realiza la recopilación de información que servirá para encontrar los parámetros del modelo, proceso conocido como calibración. A continuación se procede a calcular el equilibrio original, el cual muestra el estado de la economía antes de la aplicación de la política económica bajo análisis. El paso siguiente consiste en aplicar la política económica, lo que implica cambiar el valor de algún parámetro o variable exógena del modelo (por ejemplo: gasto público, alguna tasa impositiva o un arancel). Se calcula el nuevo equilibrio, el cual presenta el estado de la economía después de la aplicación de la política econó-

\footnotetext{
${ }^{2}$ Kehoe (2003, pp. 1-2).

${ }^{3}$ Un libro clásico que puede ser consultado es Dervis et al. (1984).
} 
FIGURA 1. Pasos para elaborar y aplicar los MEGA

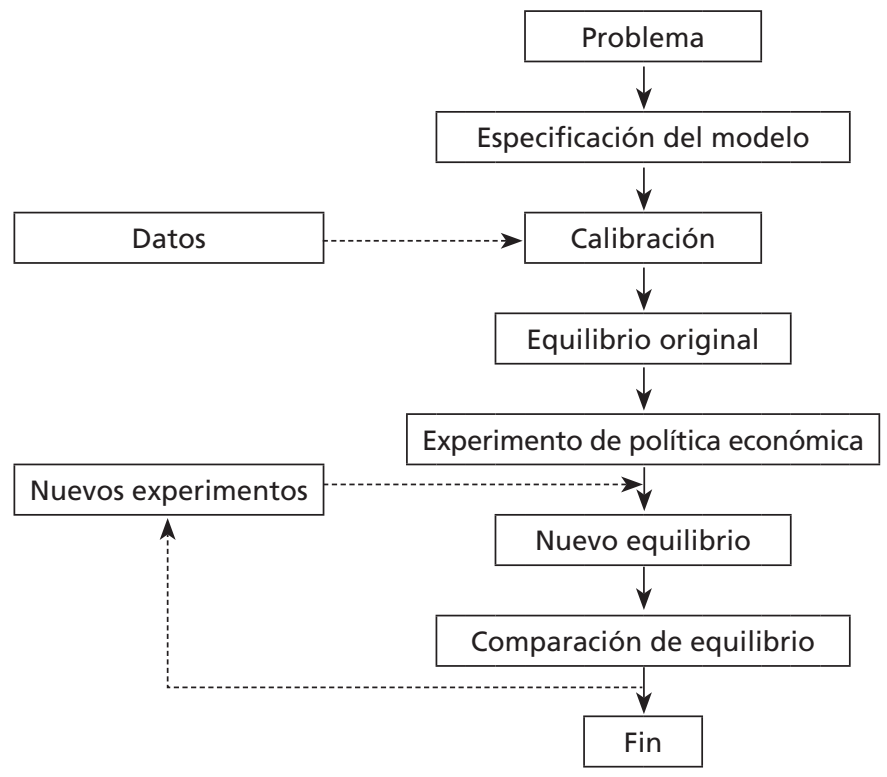

mica. Después de comparar resultados se decide si se desea realizar más experimentos; de lo contrario, el proceso concluye.

El resto del artículo presenta por sección cada uno de los pasos mencionados anteriormente y termina presentando posibles extensiones al modelo utilizado en este artículo.

\section{Problema obJeto de ESTUdio}

La motivación para la elaboración de un modelo de equilibrio general tiene su origen en la necesidad de analizar un problema económico ya existente o simplemente en el deseo de modelar una economía real, con el propósito de contar con una herramienta de análisis que tome en cuenta todas las implicaciones que en dicha economía pudiera tener cualquier cambio en materia de política económica, ya sea ésta de tipo comercial, fiscal, laboral, etcétera. En este artículo, sin embargo, no será posible analizar dichas implicaciones debido a que el principal objetivo aquí es el de familiarizar al lector con la metodología para la formulación, especificación y calibración de un MEGA. El problema que se va a analizar es el del impacto de la inversión extranjera directa (IED) en la producción y empleo sectoriales y en el nivel de bienestar. La razón para que éste sea el problema 
objeto de estudio es que puede ser analizado con el modelo sencillo utilizado en este artículo, lo cual no implica que necesariamente ésta sea la mejor metodología para analizarlo, sino que se ajusta perfectamente a los fines del presente trabajo.

\section{ESPECIFICACIÓN DEL MODELO}

Para la especificación de un modelo de equilibrio general aplicado, primero se debe tener bien claro cuáles son las características de la economía que se desea modelar. Es decir, cuántos y cuáles sectores productivos se encuentran presentes y cuáles son las posibilidades tecnológicas para cada uno de éstos; cuántos tipos de consumidores se consideran y cuáles son sus características en términos de sus preferencias y dotaciones iniciales, cuál es la relación comercial que guarda la economía con el resto del mundo, es decir, si la economía se encuentra abierta o cerrada al comercio internacional, etcétera.

\section{Entorno económico}

Por el momento debe quedarle claro al lector que para efectos del presente ejercicio no se presume la especificación del mejor modelo en términos de realismo, sino que el objetivo es ilustrar la manera en que se construye, calibra y utiliza un MEGA. Por tanto, en aras de la sencillez se supondrá la existencia de una economía cerrada, la cual se pretende modelar y cuyas características se resumen en los siguientes supuestos:

- Existen dos factores productivos: capital, $K$, y trabajo, $L$, cuya oferta es inelástica. Este supuesto implica que tanto el capital como el trabajo son homogéneos. Por simplicidad, se supone también que la producción de cada bien no requiere del uso de insumos intermedios.

- Existen dos sectores productivos, ${ }^{4}$ cada uno de los cuales produce un bien. Cada sector se comporta de manera competitiva, por lo que están integrados por muchas empresas. Supondremos que en cada sector existe una empresa representativa cuyo comportamiento equivale al del sector en su conjunto. La empresa representativa en el sector $i, i=1,2$, maximiza beneficios mediante la minimización de sus costos sujeta a un nivel dado de producción. La tecno-

\footnotetext{
${ }^{4}$ En el caso de un modelo más completo, los sectores especificados serían aquellos sobre los que existiera interés por analizar su comportamiento tras la aplicación de una determinada política económica.
} 
logía se representa mediante una función tipo Cobb-Douglas, la cual exhibe rendimientos constantes a escala. Por tanto, el problema de la empresa es:

Min

$$
T C_{i}=w L_{i}+r K_{i}
$$

s.a

$$
\bar{Y}_{i}=A_{i} L_{i}^{\beta_{i}} K_{i}^{1-\beta_{i}}
$$

en donde:

$$
\begin{aligned}
& w=\text { salario } \\
& r=\text { renta del capital } \\
& \bar{Y}_{i}=\text { producción en el sector } i \\
& L_{i}=\text { trabajo demandado en el sector } i \\
& K_{i}=\text { capital demandado en el sector } i \\
& 0<\beta_{i}<1 .
\end{aligned}
$$

El supuesto de que la función de producción sea Cobb-Douglas con rendimientos constantes a escala es sencillamente por simplicidad.

- Existe un consumidor representativo en la economía, es decir, un consumidor cuyas decisiones en cuanto a la cantidad que desea demandar de cada bien reflejan las decisiones que tiene la economía en su conjunto. ${ }^{5}$ Las preferencias del consumidor están representadas por una función de utilidad tipo CobbDouglas, ${ }^{6}$ la cual es homogénea de grado uno. En esta economía privada de un solo individuo y dos sectores productivos, el consumidor es propietario de los

${ }^{5} \mathrm{El}$ hecho de utilizar un solo consumidor responde también al propósito de formular un modelo lo más simple posible para el objeto de este artículo. Si el interés del modelador radicara en analizar cómo se vería afectada la distribución del ingreso debido a un cambio en la política económica, entonces tendrían que incluirse por los menos dos tipos de consumidores representando estratos de ingresos diferentes.

${ }^{6}$ Las formas funcionales más comúnmente utilizadas en la especificación de MEGA son las de tipo Cobb-Douglas y tipo CES. Para estas últimas es necesario conocer de antemano las respectivas elasticidades de sustitución. 
factores de producción (capital, $K$, y trabajo, $L$ ), los cuales constituyen su dotación inicial y de los cuales deriva su ingreso. Se supone, como ya es tradicional, que el comportamiento del consumidor representativo es racional y por tanto maximiza su función de utilidad-sujeto a la restricción presupuestaria que enfrenta, la cual está dada por la venta de sus servicios de trabajo y por las rentas al acervo de capital que posee. Por tanto, el problema del consumidor consiste en maximizar su función de utilidad.

$$
U\left(X_{1}, X_{2}\right)=X_{1}^{\alpha} X_{2}^{1-\alpha}
$$

sujeto a su restricción de presupuesto:

$$
P_{1} X_{1}+P_{2} X_{2}=I
$$

donde $I$ representa el ingreso del consumidor y $P_{i}$ representa el precio del bien $i, i=1,2$. Por su parte $X_{i}, i=1,2$ representa la cantidad total demandada del bien $i$. Además, $0<\alpha<1$.

- El ingreso $I$ del consumidor está dado por:

$$
I=w \bar{L}+r \bar{K}
$$

\section{Definición de equilibrio}

Una parte muy importante en la especificación del modelo es la definición de equilibrio que se va a utilizar, la cual cumple con varias funciones. En primer lugar permite ver de manera más clara el modelo que se está utilizando y, en segundo lugar, permite detectar errores en la especificación del modelo, por ejemplo, si alguna ecuación ha sido omitida.

Definición. Un equilibrio general para la economía simple que se ha planteado consiste en un vector de precios $\left(\hat{P}_{1}, \hat{P}_{2}, \hat{w}, \hat{r}\right)$ de equilibrio para los bienes y los factores, y un vector de cantidades $\left(\hat{X}_{i}, \hat{Y}_{i}, \hat{L}_{i}, \hat{K}_{i}, \hat{I}\right), i=1,2$, de demandas y ofertas de bienes, demandas de factores e ingreso del consumidor tal que dados dichos precios de equilibrio: 
- Las cantidades demandadas de cada bien $\left(\hat{X}_{1}, \hat{X}_{2}\right)$ :

$$
\begin{aligned}
& \hat{X}_{1}=\frac{\alpha \hat{I}}{\hat{P}_{1}} \\
& \hat{X}_{2}=\frac{(1-\alpha) \hat{I}}{\hat{P}_{2}}
\end{aligned}
$$

maximizan la función de utilidad del consumidor dada la restricción presupuestaria:

$$
\hat{I}=\hat{w} L+\hat{r} K
$$

- Las cantidades demandadas de factores $\left(\hat{L}_{i} \hat{K}_{i}\right)$ :

$$
\begin{aligned}
& \hat{L}_{i}=\left(\frac{\hat{Y}_{i}}{A_{i}}\right)\left(\frac{\beta_{i}}{1-\beta_{i}} \frac{\hat{r}}{\hat{w}}\right)^{1-\beta_{i}} i=1,2 \\
& \hat{K}_{i}=\left(\frac{\hat{Y}_{i}}{A_{i}}\right)\left(\frac{1-\beta_{i}}{\beta_{i}} \frac{\hat{w}}{\hat{r}}\right)^{\beta_{i}} i=1,2
\end{aligned}
$$

en cada sector $i$ minimizan los costos de cada empresa dada la restricción impuesta al nivel de producción $\hat{Y}_{i}$ y las disponibilidades tecnológicas de cada sector tal que en equilibrio las ganancias de la empresa son iguales a cero: ${ }^{7}$

$$
\hat{P}_{i} \hat{Y}_{i}=\hat{w} \hat{L_{i}}+\hat{r} \hat{K}_{i} \quad i=1,2
$$

- Los mercados de bienes se encuentran en equilibrio:

$$
\hat{X}_{i}=\hat{Y}_{i} \quad i=1,2
$$

\footnotetext{
${ }^{7}$ La razón por la cual las ganancias son de cero es porque la función de producción exhibe rendimientos constantes a escala y porque se supone que las empresas se desarrollan en un ambiente competitivo. También, dado el grado de la función de producción y el hecho de que en equilibrio cada factor recibe como pago el valor de su producto marginal, el valor del producto total se agota después de realizar dichos pagos. El modelador puede remitirse al teorema de Euler para verificarlo.
} 
- Los mercados de factores se encuentran en equilibrio:

$$
\begin{gathered}
\hat{L}_{1}+\hat{L}_{2}=\bar{L} \\
\hat{K}_{1}+\hat{K}_{2}=\bar{K} .
\end{gathered}
$$

Una vez planteado el equilibrio se puede ver con claridad que se tiene un sistema con 13 ecuaciones (6-14) y 13 incógnitas:

$$
\left(\hat{P}_{1}, \hat{P}_{2}, \hat{w}, \hat{r}, \hat{X}_{1}, \hat{Y}_{1}, \hat{L}_{1}, \hat{K}_{1}, \hat{X}_{2}, \hat{Y}_{2}, \hat{L}_{2}, \hat{K}_{2}, \hat{I}\right) \text {. }
$$

Para poder resolver este sistema son necesarias dos cosas. En primer lugar, se necesita conocer el valor de las variables exógenas $(\bar{L} y \bar{K})$ y el de los parámetros del modelo $\left(\alpha, A_{1}, A_{2}, \beta_{1}, \beta_{2}\right)$. Los valores de las variables exógenas están dados por los datos en la economía, mientras que para encontrar los valores de los parámetros del modelo se sigue un procedimiento denominado calibración, el cual se presenta en una de las secciones subsiguientes. En segundo lugar, debido a que el sistema está sobreidentificado, de acuerdo con la ley de Walras, debe indicarse de manera exógena el valor de alguna de las variables endógenas. El valor de la variable a indicarse suele ser un precio (o un índice de precios), el cual se convierte en el numerario del sistema.

\section{Ley de Walras}

La ley de Walras es muy útil porque permite verificar la consistencia del modelo. Esta ley establece que si de $\operatorname{los} n$ mercados que integran el modelo, $n-1$ se encuentran en equilibrio, entonces el mercado restante también se encuentra en equilibrio. Esto quiere decir que basta con que se cumplan 12 de las 13 ecuaciones para que la ecuación restante también lo haga, lo cual implica que el modelo está sobreidentificado y que es posible obtener múltiples soluciones. Para obtener una solución única resulta necesario establecer un numerario, es decir, el valor de uno de los precios deberá establecerse de manera exógena. Una vez hecho esto, debe tenerse claro que el valor de todas las variables nominales estará expresado en términos de este numerario. Por ejemplo, suponiendo que el precio especificado como numerario es el salario, $w$, entonces el valor de este precio será de 1 en todo momento. Por otro lado, si el precio del bien $1, P_{1}$, pasa de 1 a 1.10 , esto significa que el precio del bien 1 subió $10 \%$ respecto al salario. 
Cabe señalar que las cantidades reales no se ven afectadas por el numerario, cualquiera que éste sea, debido a que las demandas son homogéneas de grado cero en precios e ingreso, lo cual significa que al escalar los precios y el ingreso por una constante positiva, las cantidades demandadas permanecerán inalteradas.

También se puede utilizar un índice de precios como numerario, lo cual es muy apropiado si se está interesado en saber cómo varía el salario real después de algún cambio en la política económica. Si el numerario es algún índice de precios, entonces su valor será 1 en todo momento, por lo que si el salario $w$ se incrementa, entonces se puede decir que el salario real se ha incrementado. En este trabajo se supondrá que el numerario es el salario $w$.

La ley de Walras también sirve para verificar la consistencia del modelo utilizado. Si la ley no se cumple para el modelo elaborado, entonces deberá existir algún error en su especificación. Para verificar si la ley de Walras se cumple en este caso particular, se supone que se cumplen todas las ecuaciones excepto la restricción presupuestal. La ley de Walras implica que necesariamente se cumple esta última ecuación.

Sustituyendo la ecuación (12) en (11) tenemos:

$$
\hat{P}_{i} \hat{X}_{i}=\hat{w} \hat{L}_{i}+\hat{r} \hat{K}_{i} \quad i=1,2 .
$$

Sustituyendo las ecuaciones (6) y (7) en (15) tenemos:

$$
\begin{gathered}
\alpha \hat{I}=\hat{w} \hat{L}_{i}+\hat{r} \hat{K}_{1} \\
(1-\alpha) \hat{I}=\hat{w} \hat{L}_{2}+\hat{r} \hat{K}_{2} .
\end{gathered}
$$

Agregando las ecuaciones (16) y (17) tenemos:

$$
\hat{I}=\hat{w}\left(\hat{L}_{1}+\hat{L}_{2}\right)+\hat{r}\left(\hat{K}_{1}+\hat{K}_{2}\right) .
$$

Sustituyendo las ecuaciones (13) y (14) en (18) obtenemos la restricción presupuestal del consumidor.

Una vez que la especificación del modelo ha quedado bien definida se puede proceder a la obtención de los parámetros y las variables exógenas, procedimiento del cual se ocupa la sección siguiente. 


\section{CAlibración del MOdelo}

La calibración consiste en obtener datos de la economía que se está modelando con el fin de encontrar, de manera determinística, los valores de los parámetros del modelo de tal modo que éstos sean consistentes con lo observado en la economía. Así, los parámetros de los MEGA no se estiman econométricamente, sino que se calibran. Los valores de los parámetros se determinan mediante la solución del sistema algebraico de ecuaciones que representa las condiciones de equilibrio del modelo planteado. Para esto se utilizan los datos sobre precios y cantidades que caracterizan el equilibrio base o de referencia de la economía, con la idea de que los parámetros tomen valores de forma tal que la solución del modelo sea una réplica exacta de los datos compilados y éstos constituyan una solución al mismo modelo. ${ }^{8}$

Los parámetros a calibrar son: $\alpha, A_{1}, A_{2}, \beta_{1}, \beta_{2}$. Para modelos más elaborados se tendrían que calibrar otros parámetros, como la tasa de ahorro privado, tasas arancelarias, tasas impositivas y parámetros relacionados con la demanda de bienes de inversión.

El supuesto fundamental que subyace en el procedimiento de calibración es que la economía que se está modelando se encuentra en equilibrio para el periodo base seleccionado. Para el procedimiento de calibración, además de la base de datos, en ocasiones se requiere de ciertas elasticidades que deben especificarse de manera exógena, las cuales se pueden obtener a partir de estudios independientes reportados en la bibliografía o mediante estimaciones econométricas ex profeso. Entre las elasticidades que se pueden requerir, se encuentran la elasticidad de sustitución entre factores de producción, la elasticidad de sustitución entre bienes importados y bienes nacionales, ${ }^{9}$ y la elasticidad de transformación entre exportaciones y bienes nacionales..$^{10}$ En todo proceso de calibración se siguen los pasos que se presentan a continuación.

\section{Definición de cada sector}

Cada sector debe estar definido con toda claridad, lo cual ya se hizo en el momento de establecer el entorno económico y al especificar el modelo mediante

\footnotetext{
${ }^{8}$ Es requisito indispensable que el conjunto de valores de los parámetros obtenidos mediante la calibración y utilizados en el modelo sea capaz de reproducir el equilibrio observado en la economía modelada.

${ }^{9}$ En el caso de una economía abierta.

${ }^{10}$ Íbidem.
} 
las formas funcionales. Sin embargo, cabe enfatizarlo aquí con el fin de asegurarse de que todos los sectores quedarán incluidos en el sistema algebraico de ecuaciones y de que la calibración será completa. Es importante resaltar que el número de sectores depende del problema objeto de estudio. Debe evitarse incluir de manera innecesaria varios sectores, así como incluir tan pocos que el modelo no sea útil para analizar el problema objeto de estudio.

\section{Selección del año base}

El ejercicio de seleccionar el año base forma parte propiamente de la etapa de recopilación de información y corresponde al periodo que se toma como referencia para realizar los experimentos de política. Su selección es muy importante debido a que toda la información que se va a utilizar deberá corresponder a ese año particular. Uno de los criterios para elegir el año base es la posibilidad de contar con toda la información necesaria para realizar la calibración del modelo. Por ejemplo, en caso de incluir en el modelo transacciones entre sectores, se deben utilizar datos contenidos en la matriz insumo-producto, sin embargo, esta matriz se publica con poca frecuencia. En nuestro país, a partir de 1950 se han elaborado ocho matrices que corresponden a los años de 1950, 1960, 1970, 1975, 1980, 1985 y 2003, lo cual genera una restricción importante para la selección del año base.

Otro criterio para la elección del año está dado por el interés de analizar alguna política en particular. Por ejemplo, si el interés radica en analizar los impactos del Tratado de Libre Comercio (TLC) en la economía mexicana, el año base deberá ser 1993, es decir, el año anterior a la entrada en vigor de este tratado, lo cual permitirá que el modelo calibrado respecto a los datos de la economía registrados en 1993 sea capaz de reproducir los cambios registrados debido a la implantación del TLC.

\section{Sistematización de la información}

Una vez seleccionado el año base, el paso siguiente será el de recopilar y sistematizar la información en una matriz de contabilidad social (MCS). La sistematización permite garantizar que los datos estarán en condiciones de ser usados en el modelo. Dichos datos pueden ser obtenidos de diversas fuentes: cuentas nacionales, matriz insumo-producto, encuestas de ingreso y gasto de los hogares y anuarios estadísticos, por ejemplo. Asimismo, la información puede ser generada 
por diversas fuentes tales como la Secretaría de Economía (aranceles, exportaciones e importaciones, por ejemplo) y la Secretaría de Hacienda (impuestos y gasto del gobierno, por ejemplo).

Una matriz de contabilidad social (MCS) es una matriz que concentra toda la información que va a ser utilizada en el modelo. Las cuentas que aparecen como columnas también aparecen como renglones. Cada cuenta se asocia con cada una de las variables identificadas en el modelo. Por convención, los datos leídos por columna significan gastos realizados por la cuenta de dicha columna. Para el modelo que nos ocupa, se puede construir una MCS con tan sólo 7 cuentas.

La matriz utilizada para calibrar los parámetros de este modelo requiere de varios supuestos para su elaboración. Debido a que el modelo no incorpora insumos intermedios y gobierno, se supone que el valor de la producción de cada sector es igual a la suma de los pagos al trabajo y al capital. Por ejemplo, el sector 1 realiza un pago de $\$ 15$ al trabajo y $\$ 5$ al capital, por lo que el valor de su producción es de \$20. Las Cuentas Nacionales suelen presentar información sobre los pagos por concepto de sueldos y salarios. El dato que puede ser complicado encontrar es el del pago al capital. En México se genera una cuenta llamada "excedente bruto de operación", que incluye todos aquellos pagos realizados por cada sector exceptuando los sueldos y salarios, la compra de insumos intermedios y el pago de impuestos. Esta cuenta puede ser utilizada como una aproximación al pago del capital, aun cuando incluya otros pagos como rentas e intereses.

\section{CUADRO 1. Matriz de contabilidad social}

\begin{tabular}{|c|c|c|c|c|c|c|c|c|c|c|}
\hline & & & Activ & idades & & & Fact & ores & Hogares & Totales \\
\hline & & & 1 & 2 & 3 & 4 & 5 & 6 & 7 & \\
\hline & & & Sector 1 & Sector 2 & Bien 1 & Bien 2 & Trabajo & Capital & Consumidor & \\
\hline Actividades & 1 & Sector 1 & & & 20 & & & & & 20 \\
\hline & 2 & Sector 2 & & & & 20 & & & & 20 \\
\hline Bienes & 3 & Bien 1 & & & & & & & 20 & 20 \\
\hline & 4 & Bien 2 & & & & & & & 20 & 20 \\
\hline Factores & 5 & Trabajo & 15 & 10 & & & & & & 25 \\
\hline & 6 & Capital & 5 & 10 & & & & & & 15 \\
\hline Hogares & 7 & Consumidor & & & & & 25 & 15 & & 40 \\
\hline Totales & & & 20 & 20 & 20 & 20 & 25 & 15 & 40 & 160 \\
\hline
\end{tabular}

Fuente: Elaboración propia con datos creados para fines expositivos. 
Las columnas 3 y 4 del cuadro 1 muestran que cada uno de los bienes en la economía se forma con la producción del sector respectivo. Modelos más elaborados pueden incluir algunos bienes que se forman con parte o con toda la producción de algún sector más, por ejemplo, las importaciones del sector respectivo. Las columnas 5 y 6 muestran que todo el pago por salarios y capital los recibe el único consumidor que existe en la economía. Todo este ingreso lo utiliza para consumir los dos bienes que existen en la economía. Es importante resaltar que el consumo total que aparece en la columna 7 no tiene por qué coincidir con el reportado en las Cuentas Nacionales, debido a que se obtuvo de tal manera que nuestra matriz pueda estar equilibrada. Es decir, se supuso que toda la producción de cada sector la demanda el único consumidor en nuestra economía.

Lo simple que es el modelo presentado en este trabajo obliga a hacer muchos supuestos para construir la MCS. Muchos de ellos no son necesarios en modelos menos restrictivos.

\section{Precios de equilibrio iguales a uno}

Un supuesto muy importante y necesario para realizar la calibración de los parámetros y las variables exógenas es el de que en el año base la economía estuvo en equilibrio con precios iguales a uno. Esto no quiere decir que todos los precios sean iguales, sino que serán tomados como índices, de tal manera que todos sean iguales a 1 en el año base.

Este supuesto permite obtener cantidades a partir de los valores presentados en la MCS; por ejemplo, en la columna 1 el pago al trabajo igual a $\$ 15$ implica que el sector 1 utilizó 15 unidades de trabajo ya que el precio del trabajo es igual a 1. También es muy útil para poder trabajar con el modelo ya que evita que tengamos que buscar información sobre cantidades, algunas de las cuales podría ser imposible encontrar. Es muy importante tener cuidado al utilizar este supuesto. Al hacer una simulación podremos afirmar cuánto es el cambio porcentual en precios y cantidades. Por ejemplo, podremos saber en qué proporción varía la demanda de trabajo en el sector 1, pero no podemos conocer la variación en términos absolutos. Para poder hacer esto último tendríamos que saber la cantidad de trabajadores que emplea el sector 1 para aplicarle la variación porcentual obtenida por el modelo. No podemos utilizar la cantidad de 15 unidades de trabajo en el sector 1 que se desprende de la columna 1 y del supuesto de que el salario es 1, debido a que esto sólo se hace para poder hacer simulaciones con el modelo, es decir, para poder obtener las variaciones porcentuales. 


\section{Calibración de parámetros}

Para la calibración de los parámetros se hace uso de las condiciones de equilibrio, de la base de datos representada por la MCS y del supuesto de que los precios de equilibrio en el año base son iguales a 1 .

Para poder encontrar el valor de $\alpha$, se despeja su valor de la función de demanda (6):

$$
\alpha=\frac{\hat{P_{1} \hat{X}_{1}}}{I}=\frac{\hat{P_{1}} \hat{X}_{1}}{\hat{w L}+\hat{r} K}=\frac{20}{25+15}=0.5
$$

Como puede verse, para encontrar el valor de $\alpha$ sólo es necesario conocer el ingreso del consumidor y el gasto que éste realizó en el bien 1, así como su ingreso, información que se encuentra en la MCS. Encontrar el valor de $\alpha$ con base en los datos de equilibrio de referencia implica que su valor es compatible con la existencia de equilibrio en el año base.

Para encontrar el valor del parámetro $\beta_{i}$ se procede de manera similar. De las condiciones de primer orden del problema de minimización de costos de la empresa se obtiene la expresión de precios relativos de equilibrio de los factores: ${ }^{11}$

de donde se despeja $\beta_{i}$ :

$$
\frac{\hat{w}}{\hat{r}}=\frac{\beta_{i}}{1-\beta_{i}} \frac{\hat{K}_{i}}{\hat{L}_{i}}
$$

$$
\beta_{i}=\frac{\hat{w} \hat{L}_{i}}{\hat{w} \hat{L}_{i}+\hat{w} \hat{K}_{i}}
$$

por tanto, el valor de $\beta_{i}$ representa la participación de los sueldos pagados en el sector $i$ respecto al total del costo de producción en dicho sector. Utilizando la información contenida en la MCS, se tiene que: $\beta_{i}=0.75$ y $\beta_{i}=0.5$.

Continuando con la calibración de los parámetros, el valor de $A_{i}$ se encuentra despejando su valor de la función de producción, lo cual es posible puesto que en este punto ya se conoce el valor de $\beta_{i}$ y los valores correspondientes $Y_{i}$, $L_{i}$ y $K_{i}$ se encuentran disponibles en la MCS:

\footnotetext{
${ }^{11}$ Esta expresión también se obtiene dividiendo (9) entre (10).
} 


$$
A_{i}=\frac{\hat{Y}_{i}}{\hat{L}_{i}^{\beta_{i}} K_{i}^{1-\beta_{i}}} .
$$

Haciendo uso de la ecuación (21) y los datos ya mencionados se tiene que $A_{i}=1.7548$ y $A_{2}=2$.

\section{Valores de las variables exógenas}

Los valores de las variables exógenas del modelo son muy fáciles de obtener ya que éstos se encuentran contenidos en la MCS. El hecho de que el valor de los precios sea de 1 , permite interpretar las cifras correspondientes a estas variables como cantidades físicas. Así, la cantidad total de trabajo está dada por la suma de la cantidad de trabajo utilizado en el sector 1 más la cantidad de trabajo utilizada en el sector 2. Se procede similarmente para el capital. De acuerdo con la MCS de esta economía, $\bar{L}=25$ y $\bar{K}=15$.

\section{CÁlCulo del EQUilibrio ORIGiNAL}

Para obtener el equilibrio original, es decir los precios y las cantidades que garantizan un equilibrio general, se debe resolver el sistema de 13 ecuaciones y 13 incógnitas que resultó del modelo particular planteado anteriormente. Debido a que las ecuaciones no son lineales, resultaría muy problemático resolver dicho sistema de forma manual. ${ }^{12}$ Por tanto, es necesario contar con el software que permita resolver sistemas de ecuaciones no lineales. Posiblemente el lector considere que el cálculo del equilibrio original no tiene sentido alguno debido a que los precios resultarán ser iguales a 1 ya que los parámetros de las funciones de utilidad y de producción fueron calibrados partiendo de este supuesto.

En efecto, los precios que se obtendrán serán de 1, lo cual nos permitirá verificar que el modelo ha sido bien calibrado. Si el equilibrio original no reproduce las cantidades que aparecen en la base de datos de referencia utilizada para la calibración y/o si los precios resultantes de equilibrio no son iguales a uno, entonces deberá existir algún error en el procedimiento de calibración o en el programa utilizado para obtener el equilibrio, por lo que será necesario detectar y corregir el error.

\footnotetext{
${ }^{12}$ La necesidad de contar con un programa de cómputo es obvia en la medida en que el modelo se torna más completo y realista.
} 
Existen varias formas de encontrar los precios de equilibrio, algunas de las cuales son las siguientes:

- Mediante la elaboración de un programa para un algoritmo que tome en cuenta los signos de las funciones de exceso de demanda. Dicho programa puede escribirse utilizando algún lenguaje como Fortran o Matlab.

- Mediante la elaboración de un programa para un algoritmo que utilice la información sobre las derivadas de las funciones de exceso de demanda, es decir, un programa que se base en el método de Newton para resolver sistemas de ecuaciones no lineales. Este programa puede ser escrito en algún lenguaje tal como Fortran o Matlab.

- Mediante la utilización de un programa especial para resolver sistemas de ecuaciones no lineales, por ejemplo el GAMS.

- Mediante la utilización de un software especialmente diseñado para resolver modelos de equilibrio general aplicado, tal como GAMS-MPSGE.

Gran parte de los economistas que utilizan estas técnicas hacen uso del GAMS (General Algebraic Modeling System) diseñado para resolver problemas de optimización lineal y no lineal. El lector puede consultar la página www.gams. com, donde podrá descargar de manera gratuita manuales y una versión del GAMS para estudiante, ejemplos de programas elaborados para resolver MEGA, artículos sobre el tema e información sobre cursos.

\section{EXPERIMENTOS DE POLÍTICA ECONÓMICA}

Una vez concluida la calibración, el MEGA se encuentra numérica y completamente especificado para utilizarse en el análisis de política económica mediante la comparación por pares de escenarios de política con el equilibrio base de referencia. El experimento a realizar aquí es el de simular un incremento de $20 \%$ en la inversión extranjera directa que llega a esta economía. Una manera muy sencilla de modelar este incremento es aumentando $20 \%$ el acervo de capital, por lo que su nuevo valor sería de 18 . Vale la pena hacer algunos comentarios respecto a este experimento. Primero, este experimento de política económica ha sido seleccionado suponiendo que existe interés por parte del gobierno en que se dé un mayor flujo de la inversión extranjera directa (IED). Por ejemplo, algunos modelos usados para analizar los impactos económicos del TLC en los Estados Unidos y México simularon una mayor inversión extranjera exactamente de esta forma, como resul- 
tado de un relajamiento debido al tratado, de las leyes mexicanas que regulan la inversión extranjera. ${ }^{13}$

Segundo, se supone también que esta inversión llega al país y se distribuye entre los sectores de acuerdo con la demanda de capital y los precios de equilibrio. Es decir, no se supone que la nueva inversión tenga como destino algún sector en específico. Para ello se requeriría suponer que todo el capital o parte de éste es específico de algún sector. Tercero, se supone que la inversión extranjera que ha llegado al país ya se ha materializado en nuevos bienes de capital listos para producir. Por tanto, el nuevo equilibrio representa una situación que se va a presentar hasta que la inversión llegue, se compre maquinaria, equipo, edificios, etcétera y se empiece a producir con este capital.

\section{CÁlCUlo deL NUEVo EQUiLIBRio Y COMPARACIÓN CON EL ORIGINAL}

El mismo método que sirvió para obtener el equilibrio original puede ser usado para obtener el nuevo equilibrio. Lo único que se requiere modificar es el valor de $K$, de 15 a 18. El siguiente cuadro muestra los nuevos valores de equilibrio.

CUADRO 2. Comparación del nuevo equilibrio con el original

\begin{tabular}{|l|c|c|c|c|c|c|c|c|c|c|}
\hline & $Y_{1}$ & $Y_{2}$ & $P_{1}$ & $P_{2}$ & $L_{1}$ & $L_{2}$ & $w$ & $K_{1}$ & $K_{2}$ & $r$ \\
\hline Equilibrio original & 20 & 20 & 1 & 1 & 15 & 10 & 1 & 5 & 10 & 1 \\
\hline Nuevo equilibrio & 20.93 & 21.91 & 0.96 & 0.96 & 15 & 10 & 1 & 6 & 12 & 0.83 \\
\hline Variación porcentual & 4.66 & 9.54 & -4.46 & -8.71 & 0 & 0 & 0 & 20 & 20 & -16.67 \\
\hline
\end{tabular}

Fuente: Elaboración propia a partir de resultados obtenidos de la simulación realizada.

El cuadro 2 muestra que con la entrada de mayor inversión extranjera el país puede producir más. El sector que se ve más favorecido es el 2 debido a que es el más intensivo en capital, por lo que se ve muy beneficiado por la entrada al país de mayor capital y el consiguiente abaratamiento del mismo. El poder adquisitivo de los trabajadores se incrementa debido a que los precios se reducen respecto al salario (numerario); esta reducción en los precios se puede explicar por el abaratamiento del capital.

\footnotetext{
${ }^{13}$ Véase los modelos de Bachrach y Mizrahi, y Brown, Deardoff y Stern en USITC (1992).
} 
En todo experimento siempre resulta interesante analizar los cambios en el bienestar. Éstos pueden ser analizados usando la variación equivalente ( $V E$ ) y la compensada $(V C)$.

\section{La variación compensada}

La variación compensada indica el cambio en el ingreso necesario para restablecer el nivel de utilidad que tenía originalmente el consumidor.

$$
V C=I_{C}-I_{O},
$$

donde $I_{O}$ representa el ingreso original y $I_{C}$ el ingreso compensado.

De manera intuitiva, la variación compensada es la cantidad de dinero que tendría que ser dada al consumidor después de algún cambio en los precios para dejarlo "tan feliz" como estaba antes de que ocurriera cualquier cambio en ente reglón.

Para obtener expresiones que nos permitan calcular la ( $v c$ y ve), debemos obtener la función de utilidad indirecta sustituyendo las demandas de bienes en la función de utilidad. Para nuestro modelo la función de utilidad indirecta está dada por:

$$
\begin{aligned}
V\left(P_{1}, P_{2}, I\right) & =\left(\frac{\alpha I}{P_{1}}\right)^{\alpha}\left(\frac{(1-\alpha) I}{P_{2}}\right)^{1-\alpha} \\
& =I \frac{\alpha^{\alpha}(1-\alpha)^{(1-\alpha)}}{P_{1}^{\alpha} P_{2}^{(1-\alpha)}} .
\end{aligned}
$$

El ingreso que se requiere para obtener cierto nivel de utilidad está dado por:

$$
I=\frac{V P_{1}^{\alpha} P_{2}^{(1-\alpha)}}{\alpha^{\alpha}(1-\alpha)^{(1-\alpha)}} .
$$

De acuerdo con la ecuación (22), el ingreso original, tomando en cuenta que los precios son iguales a 1 , está dado por:

$$
I_{O}=\frac{V o}{\alpha^{\alpha}(1-\alpha)^{(1-\alpha)}} .
$$

El ingreso compensado, cuando los precios son $P_{1}^{*}, P_{2}^{*}$, está dado por:

$$
I_{C}=\frac{V_{O}\left(P_{1}^{*}\right)^{\alpha}\left(P_{2}^{*}\right)^{(1-\alpha)}}{\alpha^{\alpha}(1-\alpha)^{(1-\alpha)}} .
$$


Es decir, el ingreso compensado puede ser calculado a partir del ingreso original usando la siguiente ecuación:

$$
I_{C}=I_{O}\left(P_{1}^{*}\right)^{\alpha}\left(P_{2}^{*}\right)^{(1-\varepsilon)} .
$$

Sustituyendo los datos de nuestro modelo, tenemos:

$$
\begin{aligned}
I_{C} & =40(0.9554)^{0.5}(0.9129)^{0.5} \\
& =37.3563
\end{aligned}
$$

Por tanto, la $V C$ está dada por:

$$
\begin{aligned}
V C & =I_{C}-I_{O} \\
& =37.3563-40 \\
& =-2.6437 .
\end{aligned}
$$

Es decir, al consumidor se le tendrían que quitar 2.6437 pesos para que con los nuevos precios tenga el nivel de utilidad original. Esto implica que la entrada de capital mejora el nivel de bienestar del consumidor; esta mejora equivale aproximadamente a $6.6 \%$ del ingreso original.

\section{La variación equivalente}

Por otro lado, la variación equivalente indica la cantidad de dinero que tiene que ser quitada al consumidor antes de cualquier cambio en los precios para dejarlo "tan feliz" como estará después de este cambio.

$$
V E=I_{O}-I_{E},
$$

donde $I_{O}$ representa el ingreso original e $I_{E}$ el ingreso equivalente.

Intuitivamente la variación equivalente es la cantidad máxima de ingreso que el consumidor estaría deseoso de pagar para evitar cualquier cambio en los precios.

Para obtener la ve debemos de calcular el nivel de ingreso que, dados los 
140 ECONOMÍA: TEORÍA Y PRÁCTICA • Nueva Época, número 29, julio-diciembre 2008

precios originales, le permite al consumidor tener el mismo nivel de utilidad después del experimento.

El nuevo nivel de utilidad está dado por:

$$
\begin{aligned}
V^{*} & =I \frac{\alpha^{\alpha}(1-\alpha)^{(1-\alpha)}}{\left(P_{1}^{*}\right)^{\alpha}\left(P_{2}^{*}\right)^{(1-\alpha)}} \\
& =\frac{V o}{\left(P_{1}^{\alpha}\right)^{\alpha}\left(P_{2}^{*}\right)^{(1-\alpha)}}
\end{aligned}
$$

donde $V_{o}$ representa el nivel de utilidad original.

El nivel de ingreso necesario para alcanzar este nivel de utilidad, con los precios originales está dado por:

$$
\begin{aligned}
I_{E} & =\frac{V^{*} P_{1}^{\alpha} P_{2}^{(1-\alpha)}}{\alpha^{\alpha}(1-\alpha)^{(1-\alpha)}} \\
& =\frac{V_{O}}{\left(P_{1}^{*}\right)^{\alpha}\left(P_{2}^{*}\right)^{(1-\alpha)}(1-\alpha)^{(1-\alpha)}} \\
& =\frac{I_{O}}{\left(P_{1}^{*}\right)^{\alpha}\left(P_{2}^{*}\right)^{(1-\alpha)}} .
\end{aligned}
$$

Sustituyendo los datos del modelo de este capítulo, tenemos:

$$
\begin{aligned}
& I_{E} \frac{40}{(0.9554)^{0.5}(0.9129)^{0.5}} \\
& =42.83 .
\end{aligned}
$$

Por tanto, la $V E$ está dada por:

$$
\begin{aligned}
V E & =I_{O}-I_{E} \\
V E & =40-42.83 \\
& =-2.83
\end{aligned}
$$


Lo anterior significa que estando en el equilibrio original, si al consumidor se le dan 2.83 pesos obtendrá un nivel de bienestar equivalente al que tendría después del experimento. Por tanto, la ganancia en bienestar, de acuerdo con este indicador, es equivalente a $7.07 \%$ del ingreso inicial.

Además de los cambios en el nivel de bienestar, también podemos obtener los cambios en otras variables relevantes. Por ejemplo, el cambio en el producto interno bruto lo podemos obtener si evaluamos su nivel antes y después del experimento utilizando los precios del año base. Después del experimento, el PIB está dado por:

$$
\begin{aligned}
\mathrm{PIB}^{\prime} & =1(20.9327)+1(21.9089) \\
& =42.842
\end{aligned}
$$

Es decir, el PIB crece en $7.1 \%$.

\section{Posibles EXTENSIONES}

Al modelo sencillo descrito en este trabajo se le pueden hacer algunas extensiones para hacerlo más realista y apropiado para la evaluación de alguna política económica. Entre los más importantes se encuentra la incorporación de los siguientes elementos:

Gobierno. El gobierno desempeña una función muy importante en la economía, afectando de manera directa diversas variables económicas. Se puede modelar suponiendo que recibe ingresos a partir del cobro de impuestos directos e indirectos y gasta en bienes de consumo. La diferencia entre su ingreso y gasto constituirá su ahorro, el cual se puede modelar fijo o variable, según resulte más apropiado. Por tanto, el gobierno puede afectar de manera directa los precios de los bienes a partir de los impuestos indirectos, el ingreso del consumidor a partir de los impuestos directos y la demanda agregada a partir de su consumo.

Insumos intermedios. Los insumos intermedios desempeñan una función muy importante dentro de un MEGA. Cualquier cambio que presente un sector impactará de manera directa e indirecta a otros sectores a partir de las compras y ventas que se realizan entre ellos. Por ejemplo, un aumento exógeno en la demanda de algún bien incrementará no sólo la producción de dicho sector, 
sino también la producción de los sectores que le venden insumos, generándose un efecto multiplicador. De la misma manera, al aumentar la demanda de un bien, su precio puede aumentar, generando efectos en los sectores que requieren este bien como insumo. Por tanto, incorporar insumos intermedios permite apreciar no sólo los efectos que de manera directa puede tener una política económica en algunos sectores, sino también los efectos indirectos que se generan en otros sectores.

Ahorro e inversión. La existencia de ahorro permite que los agentes económicos gasten una cantidad diferente de su ingreso, lo cual abre la posibilidad de que se pueda modelar el déficit público y el de cuenta corriente, los cuales determinan la cantidad de ahorro no privado existente en la economía.

Sector externo. Incorporar este sector permite analizar problemas de política comercial. Por ejemplo, se puede analizar el impacto de imponer salvaguardas o el de llevar a cabo un acuerdo comercial.

Rendimientos crecientes y economía de escala. Diversos MEGA con competencia imperfecta y rendimientos crecientes a escala han sido utilizados para analizar impactos de política comercial. En general, se ha encontrado que cuando se toma en cuenta la existencia de rendimientos crecientes a escala, son mayores las ganancias provenientes del libre comercio. ${ }^{14}$

\section{CONCLUSIÓN}

Este trabajo busca servir como punto de partida para el conocimiento de la metodología a seguir para elaborar un MEGA. A partir de este texto, el lector podrá acceder con mayor facilidad a la bibliografía relacionada con el tema, lo cual le permitirá elaborar un modelo que incorpore varios de los elementos listados en el subtítulo anterior, con lo que podrá evaluar impactos de política económica.

\footnotetext{
${ }^{14}$ Véase Harris (1984).
} 


\section{APÉNDICE}

El sistema de ecuaciones que componen el modelo de este trabajo está conformado por las siguientes 13 ecuaciones:

$$
\begin{aligned}
\hat{X}_{1} & =\frac{0.51}{\hat{P}_{1}} \\
\hat{X}_{2} & =\frac{0.51}{\hat{P}_{2}} \\
\hat{I} & =\hat{w} 25+\hat{r} 15 \\
\hat{L}_{1} & =\left(\frac{\hat{Y_{1}}}{1.7548}\right)\left(3 \frac{\hat{r}}{\hat{w}}\right)^{0.25} \\
\hat{P_{1}} \hat{Y}_{1} & =\hat{w} \hat{L}_{1}+\hat{r} \hat{K_{1}} \\
\hat{L}_{2} & =\left(\frac{\hat{w} \hat{L}_{2}+\hat{r} \hat{K_{2}}}{2}\right)\left(\frac{\hat{r}}{\hat{w}}\right)^{0.5} \\
\hat{K}_{2} & =\left(\frac{\hat{Y_{2}}}{2}\right)\left(\frac{\hat{w}}{\hat{r}}\right)^{0.5} \\
\hat{K}_{1} & =\left(\frac{\hat{Y_{1}}}{1.7548}\right)\left(\frac{1}{3} \frac{\hat{r}}{\hat{w}}\right)^{0.75}
\end{aligned}
$$


144 ECONOMÍA: TEORÍA Y PRÁCTICA • Nueva Época, número 29, julio-diciembre 2008

$$
\begin{array}{r}
\hat{X}_{1}=\hat{Y}_{1} \\
\hat{X}_{2}=\hat{Y}_{2} \\
\hat{L}_{1}+\hat{L}_{2}=25 \\
\hat{K}_{1}+\hat{K}=15
\end{array}
$$

y 13 incógnitas:

$$
\left(\hat{P}_{1}, \hat{P}_{2}, \hat{w}, \hat{r}, \hat{X}_{1}, \hat{Y}_{1}, \hat{L}_{1}, \hat{K}_{1}, \hat{X}_{2}, \hat{Y}_{2}, \hat{L}_{2}, \hat{K}_{2}, \hat{I}\right)
$$




\section{REFERENCIAS BIBLIOGRÁFICAS}

Decaluwé, Berbard, y André Martens (1987), "Developing Countries and General Equilibrium Models: A Review of the Empirical Literature", Manuscript Report, Centro de Investigación y Desarrollo Internacional, Canadá.

Dervis, de Melo, y Robinson (1984), General Equilibrium Models for Development Policy, Nueva York, Cambridge University.

Devarajan, Shantayanan, y Sherman Robinson (2002), “The Impact of Computable General Equilibrium Models on Policy”, International Food Policy Research Institute (IFPRI).

Francois, y Shiells (comps.) (1994), Modeling Trade Policy, University Press, Cambridge.

Gutiérrez, Miguel Ángel, Francisco Venegas, y Héctor Bravo (2005), "Política fiscal en el manejo de los recursos hidráulicos: un modelo de equilibrio general computable", Estudios Económicos, Vol. 20, núm. 2, pp. 219-261.

Harris, R. (1984), “Applied General Equilibrium Analysis of Small Open Economies with Scale Economies and Imperfect Competition", American Economic Review, 74, pp. 1016-1032.

Johansen, Lief (1960), A Multisectoral Study of Economic Growth, Amsterdam, North Holland.

Kehoe, Patrick J., y Timothy J. Kehoe (1994), “A Primer on Static Applied General Equilibrium Models”, Quarterly Review, Banco de la Reserva Federal de Minneapolis, primavera.

Kehoe, Timothy J. (2003), “An Evaluation of the Performance of Applied General Equilibrium Models of the Impact of NAFTA", Research Department Staff Report 320, Banco de la Reserva Federal de Minneapolis.

Pérez, Arturo (2006), "Análisis del efecto económico de la aplicación de una medida de salvaguarda: el caso de la industria del triplay”, Economía Mexicana. Nueva Época, CIDE, Vol. XV, núm. 1, pp. 67-96.

Piggott, John, y John Whalley (1985), New Developments in Applied General Equilibrium Analysis, Cambridge University, Cambridge, Mass.

Pyatt, Graham, (1988), “A SAM Approach to Modeling”, Journal of Policy Modeling, 10(3), pp. 327-352.

Shoven, John B., y John Whalley, (1984), “Applied General-Equilibrium Models of Taxation and International Trade: An Introduction and Survey", Journal of Economic Literature, Nashville, septiembre, vol. 22, núm. 3, p. 1007.

— y - (1992), Applying General Equilibrium, Cambridge University, Cambridge, Mass. 
146 ECONOMÍA: TEORÍA Y PRÁCTICA • Nueva Época, número 29, julio-diciembre 2008

Sobarzo, Horacio (2004), "Reforma fiscal en México", Estudios Económicos, Vol. 19, núm. 2, pp. 159-180.

Srinivasan (1986), General Equilibriun Trade Policy Modeling, Londres, MIT.

Timothy J. Kehoe, y Edward C. Prescott (1995), "Introduction to the Symposium: The Discipline of Applied General Equilibrium”, Economic Theory, 6, pp. 1-11.

USITC (1992), Economy-wide Modeling of the Economic Implications of a FTA with Mexico and a NAFTA with Canada and Mexico, publication 2508, Washington. 